\title{
Ocorrência de Classes Gramaticais na Fala Espontânea de Crianças de 18 a 41 Meses com Pares
}

\author{
Occurrence of Grammatical Classes in Spontaneous Speech \\ of 18-41-Month-Old Children with their Peers
}

\author{
Andréia Schmidt*, $a$, Aline Roberta Aceituno da Costa ${ }^{b}$, \\ Michelle Cristina Norberto ${ }^{c} \&$ Anne Voss $^{c}$ \\ ${ }^{a}$ Universidade de São Paulo, Ribeirão Preto, São Paulo, Brasil, \\ ${ }^{b}$ Universidade de São Paulo, Bauru, São Paulo, Brasil \& ${ }^{c}$ Universidade Positivo, Curitiba, Paraná, Brasil
}

\begin{abstract}
Resumo
Este estudo teve como objetivo investigar a ocorrência de diferentes categorias gramaticais em 21 crianças (18 a 41 meses), interagindo espontaneamente com pares; e comparar esses dados com estudos que empregam a observação da interação adulto/criança em contexto de fala elicitada. Cada participante foi filmado durante 15 minutos, por cinco vezes (em intervalos de um mês) interagindo com colegas de classe. Os registros foram distribuídos em quatro grupos etários. As categorias gramaticais mais frequentes em todos os grupos foram verbos e substantivos. A frequência de tipos de verbos e substantivos foi equivalente entre os grupos mais jovens, mas verbos foram mais frequentes nos grupos mais velhos. Discute-se o papel do contexto de observação e do método de coleta de dados. Palavras-chave: Aquisição de linguagem, interação verbal, crianças pré-escolares.
\end{abstract}

\begin{abstract}
This study aimed to investigate the occurrences of different grammatical categories in 21 children (18 to 41 months) in situations of spontaneous speech with their peers and compare these data with studies that employ the observation of adult/child interaction in the context of elicited speech. Each child was videotaped during 15 minutes interacting with classmates. It happened for five times with intervals of one month between them. Records were joined into four groups. The most frequent grammatical categories in all age groups were verbs and nouns. The frequency of different types of verbs and nouns were equivalent among younger groups, but verbs were more frequently used in older groups. We discuss the role of observation context and the method of data sampling.

Keywords: Language acquisition, verbal interaction, preschool age children.
\end{abstract}

A aquisição da linguagem tem sido amplamente estudada por diferentes disciplinas. Recentemente, propostas sociopragmáticas do desenvolvimento cognitivo têm identificado a inserção social da criança na cultura como fator preponderante para a aprendizagem da linguagem (Nelson, 2007; Tomasello, 1999, 2003). Essas propostas têm em comum a compreensão de que fatores filogenéticos (a constituição biológica do organismo) e um mundo social estruturado (que inclui padrões de interação cultural), aliados a capacidades sociocognitivas da própria criança,

\footnotetext{
"Endereço para correspondência: Faculdade de Filosofia, Ciências e Letras de Ribeirão Preto, Departamento de Psicologia, Universidade de São Paulo, Av. Bandeirantes, 3900, Bairro Monte Alegre, Ribeirão Preto, SP, Brasil 14040-901. E-mail: aschmidt@ffclrp.usp.br Agradecimentos: As autoras agradecem à Dra. Ana Tereza Bittencourt Guimarães pelas sugestões na análise estatística dos dados, e à Dra. Ana Raquel L. Cianflone e ao Dr. Fernando Zucoloto pela leitura cuidadosa e sugestões para melhoria do trabalho.
}

viabilizam a aquisição da linguagem. Nesse sentido, o contexto interativo sociocultural e a interpretação sociocognitiva são condições essencialmente relacionais e interdependentes.

Pesquisas clássicas sobre o desenvolvimento da linguagem preocuparam-se em encontrar padrões de aquisição que poderiam ser generalizáveis entre os indivíduos. A partir desses estudos foi possível identificar uma sequência previsível de eventos, observada em várias línguas (Barrett, 1997; Befi-Lopes, Cáceres, \& Araújo, 2007; Brown, 1973; Mogford \& Bishop, 2002; Muszkat \& Mello, 2009). Um dado frequentemente discrepante nesses estudos, entretanto, é a frequência de ocorrência de palavras de diferentes classes lexicais e a sequência em que essas classes surgem no discurso de crianças de diferentes culturas. A questão que vem sendo investigada por essas pesquisas diz respeito à existência ou não de uma sequência universal na aquisição das palavras de acordo com a classe gramatical a que pertencem e os possíveis fatores facilitadores e/ou desencadeantes de tal efeito. Subjacente a estes estudos 
está a ideia de se identificar padrões gerais de aquisição da linguagem e o papel da cultura nesse processo.

Basicamente, é possível identificar na literatura duas posições distintas sobre a ocorrência ou não de uma sequência universal na aquisição do léxico, ambas apoiadas em dados empíricos. De um lado, há autores que defendem a ocorrência do predomínio da aquisição de palavras da classe gramatical dos substantivos no vocabulário das crianças, em comparação com verbos e outras categorias - o chamado viés nominal (e.g., Gentner, 1982; Golinkoff \& Hirsh-Pasek, 2006). De outro lado, diversos autores questionam a universalidade do viés nominal, baseados em dados de estudos que relativizam essa suposta preponderância (e.g., Choi \& Gopnik, 1995; Nelson, 2007; Tardif, 1996).

Golinkoff e Hirsh-Pasek (2006), autoras ligadas à primeira posição, afirmam que os substantivos são predominantes entre as primeiras 50 palavras aprendidas pelas crianças em fase de aquisição de vocabulário. Muitos estudos realizados com crianças de diferentes línguas sustentam essa afirmação, como o de Gentner (1982) com crianças falantes de Inglês, Alemão, Japonês, Kaluli, Mandarim e Turco; o de Jackson-Maldonado, Thai, Marchman, Bates e Gutierrez-Clellen (1993), sobre o vocabulário de crianças falantes de Espanhol; o de Caselli et al. (1995) com crianças falantes da língua italiana; o estudo de Bassano (2000), que investigou falantes de Francês; e o de Kim, McGregor e Thompson (2000), sobre o vocabulário de crianças falantes de Inglês e de crianças falantes de Coreano. Mais recentemente, os estudos de Bornstein et al. (2004), com crianças falantes de Espanhol, Flamengo, Francês, Hebraico, Italiano, Coreano e Inglês, e o de Imai et al. (2008), com crianças chinesas, inglesas e japonesas, também concluíram que os substantivos são mais frequentes que os verbos no discurso de crianças em fase de aquisição de vocabulário.

Crianças falantes de Português brasileiro foram investigadas por Scherer e Souza (2011), em um estudo transversal com 60 participantes entre 18 e 36 meses. Por meio do registro de suas falas quando interagiam em situação lúdica com a mãe ou a professora, as pesquisadoras levantaram a variedade (tipos) e a quantidade (ocorrências) de diferentes classes gramaticais presentes no discurso dessas crianças. Em consonância com resultados de estudos com falantes de outras línguas, as autoras verificaram que substantivos apareceram antes e com maior frequência no discurso das crianças, dados que apoiam o chamado viés nominal. Resultados similares foram obtidos com crianças brasileiras nos estudos de Bastos, Ramos e Marques (2004) e de Vidor (2008).

Autores ligados à segunda posição baseiam-se, sobretudo, em estudos realizados com falantes de línguas asiáticas e defendem que a preponderância de substantivos sobre verbos, encontrada em diversas pesquisas, pode ser atribuída a condições culturais específicas. Tardif (1996), por exemplo, estudou dez crianças de 22 meses e suas famílias, falantes de Mandarim, e observou que nove delas produziram mais tipos de verbos do que de substantivos, o que sugere que os substantivos nem sempre são aprendidos antes dos verbos. A ocorrência de mais verbos que substantivos nesse estudo pode ter ocorrido em função da situação em que os dados foram coletados (análise de recortes de situações naturais de interação entre a criança e seus familiares), o que poderia levar a um viés devido à possibilidade da interação privilegiar ou incentivar tipos específicos de palavras, de acordo com características da língua ou da cultura (Gelman \& Tardif, 1998). Outros dados dissonantes da hipótese do viés nominal também foram encontrados em pesquisas com crianças coreanas (Choi \& Gopnik, 1995; Gopnik \& Choi, 1995) e chinesas (Tardif, 1996; Tardif, Gelman, \& Xu, 1999).

Com falantes de Português brasileiro, também há dados que apoiam a hipótese da não existência de um viés nominal. O estudo conduzido por Befi-Lopes et al. (2007) investigou a fala espontânea de 60 pré-escolares com idades entre 24 e 59 meses, em interação individual com uma das pesquisadoras em contexto lúdico. A frequência de emissões de verbos foi significativamente maior que a de substantivos, até mesmo entre as crianças mais novas (24 a 35 meses). As autoras argumentaram que o fato dos participantes desta pesquisa já estarem no segundo ano de vida e, portanto, já possuírem o vocabulário em expansão, pode ter contribuído para os dados encontrados. Além disso, é possível supor que o fato das autoras analisarem a frequência de ocorrências, e não a variedade (tipos) de verbos e substantivos, o que é mais comumente analisado nas pesquisas com crianças falantes de outras línguas, pode ter influenciado os resultados. Os dados indicaram claramente, porém, que verbos são de uso corrente na fala espontânea, mesmo entre as crianças mais jovens do estudo.

Os trabalhos aqui citados apresentam dados de naturezas diversas que, se não confirmam a existência de uma espécie de viés universal para aquisição de substantivos, tampouco descartam claramente tal hipótese. A que poderia ser atribuída, então, a diferença entre os resultados apresentados?

Tardif et al. (1999) sugerem que o contexto de coleta de dados pode ter influência importante sobre os resultados produzidos. As autoras compararam a proporção de verbos e substantivos produzidos por 24 crianças falantes de Mandarim, e 24 crianças falantes de Inglês, todas com 20 meses, em três contextos diferentes de interação mãe-criança: a leitura de um livro de histórias, uma situação lúdica com um conjunto de brinquedos "mecânicos" (que tinham movimento) e uma situação de brincadeira com brinquedos "neutros" (sem uma função definida). Os dados indicaram que o contexto de leitura de livro de histórias favoreceu a maior emissão de substantivos que de verbos, independente da língua, tanto para as mães quanto para as crianças. Já o contexto de brincadeira com brinquedos (mecânicos ou neutros), favoreceu a maior emissão de verbos pelas mães, mas uma emissão equivalente de verbos e substantivos pelas crianças; entretanto, a maioria das crianças falantes de Mandarim apresentou um número 
Schmidt, A., Costa, A. R. C., Norberto, M. C. \& Voss, A. (2014). Ocorrência de Classes Gramaticais na Fala Espontânea de Crianças de 18 a 41 Meses com Pares.

maior de verbos que de substantivos. Dados semelhantes foram encontrados por Ogura, Dale, Yamashita, Murase e Mahieu (2006) em estudo com crianças japonesas e suas mães. Esses resultados sugerem que o contexto da coleta pode exercer influência nos dados produzidos.

Outro fator importante a ser considerado nos estudos sobre aquisição de categorias lexicais por crianças é o método empregado (Bastos et al., 2004) que, em geral, baseia-se: (a) na coleta de informações com os cuidadores sobre o vocabulário das crianças (diários); (b) na construção de listas de checagem de palavras ou em testes de evocação de palavras; e (c) na observação da interação entre crianças e adultos, geralmente em situações controladas, ainda que em contextos lúdicos.

Várias limitações podem ser levantadas sobre esses métodos. O preenchimento de diários por mães depende não apenas de sua memória, mas também da importância dada por elas para a ocorrência de certas palavras no vocabulário da criança; listas de checagem, por não serem exaustivas, podem não contemplar adequadamente $o$ vocabulário dos participantes (Bornstein et al., 2004), ou podem indicar o uso da palavra sem que a criança de fato apresente o domínio completo do seu significado (Imai et al., 2008). Medidas observacionais também podem ser enviesadas, não apenas pelo contexto em que ocorre a coleta de dados (Tardif et al., 1999), mas também porque podem produzir amostras pouco representativas do vocabulário dos participantes, como no caso de crianças que se intimidam diante de observadores não familiares (Bornstein et al., 2004; Pine, Lieven, \& Rowland, 1996). Além disso, é preciso considerar que a situação de interação entre criança e adulto (como aquelas empregadas geralmente nas pesquisas, em que o contexto de coleta de dados é de fala elicitada) traz, em si, características que podem afetar fortemente a produção de fala pela criança em função de demandas específicas do adulto sobre o seu desempenho.

Pessoa e Seidl de Moura (2011) realizaram um estudo longitudinal da interação entre quatro díades mãe-bebê, analisando as características das falas das mães dirigidas aos seus bebês, quando estes tinham entre 13 e 24 meses. A fala das mães foi classificada em relação a aspectos sintáticos e semânticos. Dentre os resultados apresentados, destaca-se a análise dos aspectos sintáticos da fala das mães: cerca de metade das sentenças proferidas por elas na situação estudada eram afirmativas, descrevendo situações relacionadas ao contexto próximo e ao bebê; em média, entre 20 e $25 \%$ das sentenças eram interrogativas, e entre 12 e $27 \%$ eram imperativas. Esses dados apontam que, nas díades estudadas, cerca de um terço da fala da mãe dirigida ao bebê tinha como efeito a tentativa de engajamento da criança em atividades específicas (sentenças imperativas e interrogativas), de natureza motora ou verbal. O estudo não descreve a produção de fala das crianças, mas a análise dos dados da produção da mãe sugere o quanto, na situação estudada, a mãe pode demandar desempenhos específicos dos bebês. Tal relação é apoiada por estudo de Goldfield (1993), que observou a interação entre crianças de um ano e suas mães, em contexto com e sem brinquedo. A frequência de substantivos apresentados pelas mães variou nos diferentes contextos, mas nas situações de observação, as mães instavam seus filhos a produzir mais substantivos que verbos.

Os dados sobre a influência das variáveis de contexto, assim como os que indicam que o foco da fala dirigida à criança pode influenciar sua produção, sugerem que é preciso ter cuidado na análise dos resultados de estudos que empregam como método de investigação situações de interação adulto-criança. Será que a proporção das diferentes classes gramaticais no vocabulário de crianças difere quando se analisa a interação natural delas com seus pares? Nessas condições, o contexto natural de brincadeiras favoreceria a ocorrência maior de uma ou outra categoria gramatical?

O objetivo do presente trabalho foi investigar, por meio de um estudo de seguimento, a frequência de ocorrências de diferentes categorias gramaticais (verbo, substantivo, adjetivo, advérbio, pronome, onomatopeia, interjeição, artigo, conjunção, preposição e numeral) em crianças em fase de aquisição inicial da Língua Portuguesa, com idades entre 18 e 41 meses, em situação de fala espontânea com seus pares. Além disso, procurou-se comparar os dados relativos à ocorrência dessas classes gramaticais na presente pesquisa, com pesquisas que empregam a observação da interação entre adulto e criança em contexto de fala elicitada.

A faixa etária foi definida levando-se em consideração o que a literatura apresenta sobre o desenvolvimento da linguagem infantil. Golinkoff e Hirsh-Pasek (2006) apontam que entre os 16 e os 24 meses os bebês começam a mapear palavras e seus referentes (ações, objetos ou propriedades de objetos e eventos). Além disso, sugerem que é a partir dos 24 meses que se pode observar que as frases produzidas são completas, apresentando maior variedade de estruturas. Assim, o estudo partiu dos 18 meses, período em que a produção de fala já pode ser observada e quando os eventos acústicos ligam-se a eventos ambientais, e seguiu até os 41 meses, quando as sentenças já devem ser completas e a criança já é capaz de interpretar significados complexos.

\section{Método}

\section{Participantes}

Participaram da pesquisa 21 crianças (nove meninos e 12 meninas), com idades entre 18 e 36 meses no início da coleta de dados (no último registro de dados, as mais velhas tinham 41 meses). Todas frequentavam uma escola particular de Educação Infantil em período integral (ver Tabela 1), e eram provenientes de famílias de nível socioeconômico médio. Todas foram avaliadas pelo Teste de Triagem de Desenvolvimento Denver II (Frankenburg, Dodds, Archer, Shapiro, \& Bresnick, 1992) e não apresentaram indicativos de atraso no desenvolvimento. A participação no estudo foi autorizada pelos pais, que assinaram um Termo de Consentimento Livre e Esclarecido. A filmagem das 
crianças foi realizada predominantemente nas salas de aula das crianças durante a realização de atividades recreativas livres, que eram monitoradas pelos professores, mas que não sofriam interferência destes. Nessas atividades, as crianças poderiam brincar com objetos diversos (blocos de montar, bonecos, miniaturas de animais, etc.). Eventualmente, essas atividades ocorriam no parque da escola, local onde havia areia e equipamentos para as crianças (escorregador, balanços, carrossel, etc.).

Tabela 1

Informações Gerais Sobre os Participantes do Estudo

\begin{tabular}{cccccccc}
\hline Partic. & Sexo & $\begin{array}{c}\text { Idade } \\
\text { Inicial } \\
\text { (meses) }\end{array}$ & $\begin{array}{c}\text { Idade } \\
\text { Final } \\
\text { (meses) }\end{array}$ & Partic. & Sexo & $\begin{array}{c}\text { Idade } \\
\text { Inicial } \\
\text { (meses) }\end{array}$ & $\begin{array}{c}\text { Idade } \\
\text { Final } \\
\text { (meses) }\end{array}$ \\
\hline P1 & M & 18 & 24 & P12 & F & 30 & 35 \\
P2 & M & 20 & 26 & P13 & F & 30 & 35 \\
P3 & F & 21 & 26 & P14 & M & 30 & 35 \\
P4 & F & 20 & 26 & P15 & M & 30 & 35 \\
P5 & F & 20 & 26 & P16 & F & 30 & 35 \\
P6 & M & 22 & 27 & P17 & F & 31 & 37 \\
P7 & M & 22 & 28 & P18 & M & 33 & 38 \\
P8 & F & 23 & 28 & P19 & M & 34 & 39 \\
P9 & F & 25 & 31 & P20 & F & 35 & 40 \\
P10 & M & 27 & 33 & P21 & F & 36 & 41 \\
P11 & F & 28 & 33 & & & & \\
\hline
\end{tabular}

\section{Procedimento de Coleta de Dados}

A pesquisa foi aprovada pelo Comitê de Ética em Pesquisa da Universidade Positivo (Protocolo 128/2009). Após o consentimento da escola e dos pais, e um período de habituação entre as pesquisadoras e as crianças, foram iniciadas as filmagens propriamente ditas que ocorreram durante atividades de brincadeira livre, diariamente, por aproximadamente uma hora. Os critérios para escolha das crianças a serem filmadas a cada dia eram o seu engajamento em uma atividade com, no mínimo, mais uma criança, e ter se passado pelo menos um mês desde o seu último registro. Cada criança era focalizada individualmente durante 15 minutos consecutivos; concluído esse tempo, passava-se a filmar outra criança, e assim sucessivamente, até que a atividade livre fosse interrompida pela professora. Cada criança foi filmada cinco vezes, com intervalo de aproximadamente um mês entre os registros.

\section{Procedimento de Análise dos Dados}

As filmagens das crianças foram distribuídas para análise em quatro grupos, de acordo com a faixa etária da criança no momento do registro: G1, filmagens de crianças com idade entre 18 e 24 meses; G2, filmagens de crianças de 25 a 30 meses; G3, filmagens de crianças entre 31 e 36 meses; e G4, filmagens de crianças entre 37 e 41 meses (ver Tabela 2). Desta forma, uma criança cuja primeira filmagem ocorreu quando tinha 21 meses, por exemplo, e a última quando tinha 26 meses, teve três de seus registros computados no G1 e dois no G2. Portanto, o $n$ de cada grupo não se refere ao número de indivíduos, mas sim ao número de filmagens analisadas no grupo.

Tabela 2

Número de Filmagens de Meninas e Meninos, de Acordo com a Faixa Etária dos Participantes no Momento dos Registros

\begin{tabular}{ccccc}
\hline Grupo & $\begin{array}{c}\text { Idade } \\
\text { (meses) }\end{array}$ & $n$ meninas & $n$ meninos & Total \\
\hline 1 & 18 a 24 & 12 & 13 & 25 \\
2 & 25 a 30 & 17 & 12 & 29 \\
3 & 31 a 36 & 23 & 15 & 38 \\
4 & 37 a 41 & 8 & 5 & 13 \\
\hline
\end{tabular}

Todas as filmagens foram transcritas na íntegra, incluindo as ações motoras das crianças, suas falas/vocalizações e o contexto da atividade em curso. Em seguida, as falas/vocalizações das crianças foram classificadas de acordo com as seguintes classes gramaticais: verbo, subs- 
Schmidt, A., Costa, A. R. C., Norberto, M. C. \& Voss, A. (2014). Ocorrência de Classes Gramaticais na Fala Espontânea de Crianças de 18 a 41 Meses com Pares.

tantivo, adjetivo, pronome, advérbio, interjeição, artigo, conjunção, preposição, numeral e onomatopeia.

Os dados referentes ao número de vocalizações por minuto realizadas por meninas e meninos, foram avaliados quanto ao padrão de distribuição de dados por meio do teste de Lilliefors. Uma vez verificada a distribuição normal dos dados, os mesmos foram comparados entre gêneros e faixas etárias por meio do teste ANOVA fator duplo.

As frequências de vocalizações por classes lexicais não se encontravam em distribuição normal, logo as análises foram realizadas por meio de testes não paramétricos. Ao comparar as frequências das vocalizações por classe gramatical e por faixa etária, foi utilizado o teste de qui quadrado para bondade de ajustamento. A comparação entre as frequências absolutas de vocalizações das classes verbo e substantivo foi realizada por meio do teste não paramétrico Mann-Whitney- $U$. A comparação das classes lexicais entre as faixas etárias em cada um dos gêneros foi realizada por meio do teste de Kruskal-Wallis, seguida da comparação múltipla das categorias pelo método de Dunn.

Os dados referentes às frequências de ocorrência e de tipos de substantivos e verbos foram comparados entre os grupos etários por meio do teste ANOVA-fator único. Em situações de evidência de significância estatística, os dados foram submetidos ao teste de acompanhamento Least Square Difference. As frequências de ocorrências, assim como as frequências de tipos de substantivos e verbos foram comparadas entre si, para cada um dos grupos isoladamente, por meio do teste $t$ para amostras pareadas. Em todos os testes foi utilizado o nível de significância de 0,05 .

\section{Resultados}

Ao analisar a frequência de vocalizações por minuto das crianças, não foram verificadas diferenças estatísticas significativas entre os gêneros e as faixas etárias, contudo meninas e meninos apresentaram a mesma tendência de aumento do número de vocalizações com o passar do tempo $\left(F_{3,97}=1,90 ; p=0,13\right)$. Ao comparar cada faixa etária entre os gêneros, verificou-se que apenas no grupo de 31 a 36 meses de idade (G3) o número de vocalizações emitidas pelos meninos foi significativamente menor que das meninas $(p<0,05)$. Entre as meninas, a cada grupo etário há um aumento significativo do número de vocalizações $(p<$ $0,05)$. Entre os meninos, porém, o aumento do número de vocalizações só é significativo no grupo de 37 a 42 meses $(p<0,05)$, sendo todos os grupos anteriores considerados semelhantes estatisticamente $(p>0,05)$.

A Tabela 3 apresenta as ocorrências das classes lexicais mais frequentemente emitidas por meninos e meninas dos diferentes grupos etários (verbo, substantivo, pronome, advérbio, adjetivo, onomatopeia e interjeição). A frequência de emissão de artigos, conjunções, preposições e numerais foi muito baixa para todas as faixas etárias estudadas. Dessa forma, a análise dos dados será apresentada apenas em relação às classes gramaticais com maior frequência de emissão.

As ocorrências de verbos foram significativamente mais frequentes em todas as faixas etárias e em ambos os sexos $(p<0,05)$. A segunda classe gramatical mais frequente, 'substantivo', foi significativamente superior em todas as faixas etárias das meninas $(p<0,05)$, contudo semelhante às demais categorias, exceto verbo, nas faixas etárias de 18 a 24 meses e de 37 a 42 meses $(p>0,05)$. Comparando-se apenas as classes 'verbo' e 'substantivo', a frequência de verbos foi significativamente superior, realizando-se a análise independente da faixa etária ou do sexo $(U=4189 ; p=0,003)$.

A frequência de vocalizações das meninas da categoria 'pronome' aumentou significativamente a partir dos 31 meses de idade $\left(\mathrm{H}_{3,60}=23,76 ; p<0,01\right)$. Já entre os meninos, observa-se a elevação da frequência de vocalizações de pronomes, contudo esse aumento não é significativamente diferente entre as faixas etárias $\left(\mathrm{H}_{3,44}=4,51 ; p=0,21\right)$.

Ao avaliar a frequência de emissões de advérbios entre as meninas, foram observadas diferenças estatísticas significativas $\left(\mathrm{H}_{3,60}=25,03 ; p<0,01\right)$ sendo possível verificar que, a partir dos 31 a 36 meses, ocorreu um aumento significativo de tais emissões. Já entre os meninos, não foi possível observar este aumento significativo $\left(\mathrm{H}_{3,45}=\right.$ $4,19 ; p=0,24)$, apesar de ser detectada uma tendência ao aumento a partir dos 37 meses de idade.

Comparando-se as frequências de emissões de adjetivos entre as meninas, não foram verificadas diferenças estatísticas significativas $\left(\mathrm{H}_{3,60}=4,41 ; p=0,22\right)$, apesar de ser observada uma tendência ao aumento de tais emissões ao longo das faixas etárias estudadas. Entre os meninos, também não foram observadas diferenças estatísticas entre os grupos etários $\left(\mathrm{H}_{3,45}=4,51 ; p=0,21\right)$, não havendo tendência de elevação da frequência de emissões de adjetivos entre os grupos.

Ao comparar a frequência de emissões de onomatopeias, foi possível verificar a tendência maior de vocalização por parte dos meninos na faixa etária entre 25 e 36 meses em relação às demais faixas etárias $(p<0,05)$, o que parece ser justificável pelo contexto de brincadeiras típicas de meninos (brincadeiras com carrinhos e de lutar, por exemplo). As meninas apresentaram frequências equivalentes dessa categoria em todas as faixas etárias analisadas $(p>0,05)$.

Finalmente, ao analisar a frequência de emissões de interjeições por parte das meninas, há diferenças estatísticas significativas $\left(\mathrm{H}_{3,60}=11,89 ; p=0,007\right)$, observando-se o aumento das emissões principalmente a partir dos 37 a 42 meses de idade. Entre os meninos, não foi possível verificar diferenças significativas $\left(\mathrm{H}_{3,45}=5,69 ; p=0,12\right)$, contudo, observa-se a mesma tendência verificada entre as meninas.

Considerando que as classes lexicais mais frequentes em todos os grupos estudados foram as de verbo e substantivo, realizou-se uma análise comparativa de ocorrências e tipos dessas duas classes, como mostra a Tabela 4. 
Psicologia: Reflexão e Crítica, 27(3), 573-581.

Tabela 3

Frequência Relativa Percentual (\%) e Absoluta (FA) de Ocorrências das Categorias Lexicais nas Diferentes Faixas Etárias, para Meninos e Meninas

\begin{tabular}{|c|c|c|c|c|c|c|c|c|c|}
\hline & \multirow[b]{3}{*}{$\mathrm{F}$} & $\%$ & FA & $\%$ & FA & $\%$ & FA & $\%$ & FA \\
\hline \multirow[b]{2}{*}{ G1 } & & \multicolumn{2}{|c|}{ Verbos } & \multicolumn{2}{|c|}{ Substantivos } & \multicolumn{2}{|c|}{ Pronomes } & \multicolumn{2}{|c|}{ Advérbio } \\
\hline & & 40 & 44 & 26 & 29 & 3 & 3 & 5 & 6 \\
\hline & $\mathrm{M}$ & 38 & 55 & 18 & 26 & 12 & 18 & 17 & 25 \\
\hline \multirow[t]{2}{*}{$\mathrm{G} 2$} & $\mathrm{~F}$ & 35 & 189 & 28 & 151 & 12 & 63 & 8 & 43 \\
\hline & $\mathrm{M}$ & 26 & 75 & 20 & 57 & 15 & 43 & 6 & 17 \\
\hline \multirow[t]{2}{*}{ G3 } & $\mathrm{F}$ & 32 & 342 & 23 & 239 & 15 & 158 & 12 & 122 \\
\hline & M & 36 & 107 & 21 & 62 & 14 & 42 & 10 & 30 \\
\hline \multirow[t]{3}{*}{ G4 } & $\mathrm{F}$ & 36 & 198 & 24 & 132 & 13 & 72 & 10 & 54 \\
\hline & $\mathrm{M}$ & 31 & 65 & 17 & 35 & 18 & 37 & 11 & 24 \\
\hline & & \multicolumn{2}{|c|}{ Adjetivo } & \multicolumn{2}{|c|}{ Onomatopeia } & \multicolumn{2}{|c|}{ Interjeição } & & \\
\hline \multirow[t]{2}{*}{ G1 } & $\mathrm{F}$ & 3 & 3 & 7 & 8 & 13 & 14 & & \\
\hline & M & 6 & 9 & 4 & 6 & 3 & 4 & & \\
\hline \multirow[t]{2}{*}{$\mathrm{G} 2$} & $\mathrm{~F}$ & 2 & 12 & 3 & 14 & 2 & 10 & & \\
\hline & $\mathrm{M}$ & 7 & 30 & 16 & 46 & 6 & 16 & & \\
\hline \multirow[t]{2}{*}{ G3 } & $\mathrm{F}$ & 3 & 27 & 1 & 7 & 4 & 42 & & \\
\hline & $\mathrm{M}$ & 1 & 3 & 14 & 41 & 4 & 13 & & \\
\hline \multirow[t]{2}{*}{ G4 } & $\mathrm{F}$ & 3 & 16 & 0 & 0 & 4 & 23 & & \\
\hline & $\mathrm{M}$ & 1 & 3 & 2 & 5 & 13 & 14 & & \\
\hline
\end{tabular}

Tabela 4

Média e Desvio Padrão de Tipos e Ocorrências de Verbos e Substantivos nos Diferentes Grupos Etários

\begin{tabular}{rccccccc}
\hline & \multicolumn{5}{c}{ Substantivos } & \multicolumn{5}{c}{ Verbos } \\
\cline { 2 - 8 } & Total & Média & $D P$ & Total & Média & $D P$ & $p$ \\
\hline Ocorrências & & & & & & & \\
G1 & 55 & 2,3 & 3,1 & 99 & 4,1 & 3,7 & 0,04 \\
G2 & 208 & 7,2 & 7,5 & 264 & 9,1 & 8,8 & 0,05 \\
G3 & 301 & 7,9 & 9,6 & 449 & 11,8 & 10,9 & 0,01 \\
G4 & 167 & 12,8 & 10,2 & 263 & 20,2 & 13,1 & 0,01 \\
Tipos & & 0,001 & & & 0,18 & & \\
& & & & & & & \\
G1 & 36 & 2,8 & 4,4 & 24 & 2,5 & 2,6 & 0,65 \\
G2 & 43 & 2,9 & 3,5 & 27 & 3,2 & 2,3 & 0,59 \\
G3 & 80 & 3,8 & 3,0 & 61 & 5,4 & 4,1 & 0,01 \\
G4 & 58 & 6,8 & 4,9 & 47 & 7,9 & 5,1 & 0,09 \\
\hline & & 0,009 & & & 0,0002 & & \\
\hline
\end{tabular}


Schmidt, A., Costa, A. R. C., Norberto, M. C. \& Voss, A. (2014). Ocorrência de Classes Gramaticais na Fala Espontânea de Crianças de 18 a 41 Meses com Pares.

Sobre as ocorrências de substantivos, foram verificadas diferenças estatísticas entre os grupos $\left(F_{3,101}=5,47 ; p=\right.$ $0,001)$. O G1 difere dos demais grupos, pois apresenta média significativamente mais baixa $(p<0,05)$. Os demais grupos são considerados estatisticamente equivalentes $(p$ $>0,05)$, com exceção do G4 que apresenta média significativamente mais elevada que o G2 $(p<0,05)$.

Ao comparar as frequências de ocorrências de substantivos e verbos em cada um dos grupos, foi possível verificar que a ocorrência de verbos é significativamente superior $(4,1+3,7)$ à ocorrência de substantivos $(2,3 \pm 3,1)$ no G1 $(t=2,18 ; p=0,03)$. Estas diferenças significativas também foram observadas nos demais grupos. No G2, a média de ocorrência de verbos foi significativamente superior a de substantivos $(t=-2,02 ; p=0,05)$, assim como no G3 $(t=$ $-2,95 ; p=0,005)$ e no $\mathrm{G} 4(t=-5,18 ; p<0,01)$, como se pode observar na Tabela 4.

$\mathrm{Na}$ análise da frequência dos tipos de substantivos, foi constatada diferença estatística entre os grupos $\left(F_{3,101}=\right.$ $4,00 ; p=0,009)$. O G4 difere dos demais grupos, pois apresenta média significativamente mais elevada $(p<0,05)$. Quando avaliada a frequência dos tipos de verbos, também se observam diferenças estatísticas significativas $\left(F_{3,101}=\right.$ $9,07 ; p<0,01)$. Todos os grupos apresentam frequências médias diferentes entre si $(p<0,05)$, com exceção dos grupos 1 e 2, que apresentam médias equivalentes $(p>0,05)$.

Quando comparadas as duas categorias lexicais (verbos e substantivos) nos respectivos grupos, foi possível verificar que as frequências de tipos são equivalentes nos grupos 1 e $2(t=0,45 ; p=0,65 ; t=-0,54 ; p=0,59)$. Contudo, no G3 é evidente a maior frequência de tipos de verbos $(5,4 \pm$ $4,1)$ quando comparada à frequência de tipos de substantivos $(3,8 \pm 3,0 ; t=-4,62 ; p<0,01)$. Observa-se também a mesma tendência no $\mathrm{G} 4$, apesar dessa diferença não ser considerada estatisticamente significativa $(t=-1,85 ; p=$ 0,09), conforme Tabela 4.

\section{Discussão}

As classes gramaticais com maior frequência de ocorrência no vocabulário inicial das crianças deste estudo foram verbos, substantivos, advérbios, adjetivos, onomatopeias e interjeições; dentre essas, as mais frequentes foram verbos e substantivos, que têm sido apontadas por inúmeros estudos como as classes mais frequentemente emitidas por crianças falantes de diferentes línguas (e.g., Gentner, 1982; Golinkoff \& Hirsh-Pasek, 2006; Kauschke \& Hofmeister, 2002). Interessante notar, no entanto, que ainda que verbos e substantivos tenham sido as categorias mais frequentes em todos os grupos, juntas elas representaram pouco mais da metade do vocabulário registrado, indicando que a aprendizagem de palavras pelas crianças (mesmo as mais novas) ocorre para além da "rota referencial", e provavelmente depende de uma série de variáveis familiares e culturais (Nelson, 2007). Os dados do presente trabalho também indicaram que a variável sexo não foi relevante para a frequência de ocorrência das categorias gramaticais analisadas, e que, conforme o esperado, pode-se observar o aumento na frequência de ocorrências da maioria das classes estudadas em função da idade, da mesma forma que em outros estudos com crianças falantes de Português brasileiro (Bastos et al., 2004; Befi-Lopes et al., 2007; Scherer \& Souza, 2011).

Os principais dados de interesse do presente artigo referem-se à análise de ocorrências e de tipos de verbos e substantivos produzidos por crianças de diferentes faixas etárias na sua fala espontânea com pares. Observou-se que, na situação estudada, verbos foram mais frequentes, tanto na análise das ocorrências como dos tipos emitidos, ainda que nem sempre a diferença encontrada tenha sido estatisticamente significativa. Esses dados contrastam com os de estudos internacionais (e.g., Bornstein et al., 2004; Caselli et al., 1995; Gentner, 1982; Imai et al., 2008; Jackson-Maldonado et al., 1993), mas também diferem de dados de estudos com crianças brasileiras, como os de Scherer e Souza (2011) e de Vidor (2008). Scherer e Souza, ao estudar uma amostra transversal de crianças entre $18 \mathrm{e}$ 32 meses, encontraram que para as mais jovens, na faixa dos 18 meses, a classe dos substantivos foi dominante, mas que na faixa dos 24 e dos 32 meses, os verbos tendiam a se igualar (24 meses) ou a superar (32 meses) os substantivos, tanto na análise de tipos quanto na de ocorrências. O estudo longitudinal de Vidor, com quatro crianças entre 12 e 36 meses, mostrou dados semelhantes: predominância de substantivos na fase inicial de aquisição do léxico, até cerca de 24 meses, com diminuição dessa diferença entre os dois e três anos; no entanto, analisando os dados de ocorrências, a autora encontrou uma predominância apenas discreta de substantivos em relação a verbos até cerca de 20 meses, com tendência à estabilização da relação após essa idade.

A que se pode atribuir essas diferenças? A hipótese mais provável diz respeito à situação de coleta dos dados. Estudos internacionais empregam, em geral, inventários de linguagem, listas de verificação e diários preenchidos pelos responsáveis das crianças estudadas. Esses instrumentos apresentam inúmeras limitações, apontadas por diversos autores e descritas nesse estudo (Bastos et al., 2004; Bornstein et al., 2004; Pine et al., 1996; Stoll et al., 2012). Em função dessas limitações, autores como Bastos et al. (2004) afirmam que dados coletados em situação dialógica ou de interação podem refletir melhor o vocabulário das crianças, por serem mais próximas do contexto natural de aprendizagem e uso da linguagem.

É importante considerar, porém, que esse tipo de situação também pode constituir um viés. Pesquisas que empregam essa metodologia costumam registrar dados da interação entre adultos e crianças, em situação natural (e.g., Bastos et al., 2004; Scherer \& Souza, 2011) e em contexto de elicitação, ou seja, em que o pesquisador provoca a interação com a criança por meio de perguntas ou pelo engajamento em brincadeira conjunta com ela (Befi-Lopes et al., 2007; Vidor, 2008). Nesses casos, é preciso considerar que diferentes contextos de interação criança-adulto podem favorecer a produção de substanti- 
vos ou de verbos, tanto por parte das crianças, quanto dos adultos, independente da língua falada pelos participantes (Choi, 2000; Goldfield, 1993; Ogura et al., 2006; Tardif et al., 1999). Há indícios, por exemplo, de que há situações em que, ao interagir com os adultos, as crianças podem produzir mais substantivos que verbos "sob demanda" (Tardif et al., 1999), ou seja, em função das falas dirigidas a elas pelo adulto (perguntas ou comentários, por exemplo), mais orientadas para a ação ou para a nomeação de objetos (Pessoa \& Seidl de Moura, 2011).

No caso da metodologia empregada nessa pesquisa, em função do foco não ser a interação adulto-criança, mas a interação entre pares, não parece ter havido uma demanda linguística específica criada pela situação de coleta de dados que, inclusive, não foi estruturada especificamente para esse fim. No contexto estudado, as interações pareceram se caracterizar pela horizontalidade nas relações, que propiciou a ampla expressão do repertório gramatical das crianças, fornecendo condições para que elas desempenhassem um papel ativo umas em relação às outras. $O$ desenvolvimento da linguagem pressupõe, como afirma Tomasello (1999), a participação da criança em atividades comunicativas (em contextos de atenção compartilhada com o adulto, a princípio), a partir das quais irá aprender a entender os diferentes papeis que falantes e ouvintes desempenham na atividade. A partir disso, ela será capaz de expressar e desempenhar, em situações diferentes e em relação a outras pessoas, as mesmas "intenções" comunicativas que foram dirigidas a ela. Nesse sentido, talvez, na falta de uma demanda específica do adulto, as crianças tenderam a empregar uma variedade maior de verbos em comparação com substantivos exatamente porque a situação lúdica era propícia para tal: um convite para a ação, para o brincar conjunto, ou uma oportunidade para a descrição da própria ação e da ação do outro, o que, sem dúvida, pode caracterizar essa situação como privilegiada para a exploração do potencial gramatical das crianças.

Contextos de interação que não privilegiam a relação da criança com o adulto são pouco pesquisados. Além disso, por ser este um estudo de seguimento, o fato da maior produção de verbos ocorrer para todas as faixas estudadas, resultado diferente dos estudos com crianças brasileiras já citados, sugere a necessidade de aprofundamento sobre o tipo de input linguístico que as crianças oferecem umas para as outras em situação de interação. No entanto, deve-se considerar, também, as limitações da presente pesquisa. A curta duração dos registros em vídeo (15 minutos), o espaçamento entre eles (cerca de um mês) e a homogeneidade da amostra em relação a condições socioeconômicas não permitem generalizações dos dados para além dos participantes estudados. Tal generalização demandaria estudos de seguimento com amostras maiores, que contemplassem crianças de diferentes condições socioculturais e com registros mais longos e menos espaçados. Além disso, o aprofundamento da análise das falas das crianças, incluindo uma descrição mais ampla do contexto lúdico em que ocorreram e de sua funcionalidade para atender às demandas do ambiente, poderia trazer dados ricos sobre a aquisição do léxico em condições naturais. Estudos futuros devem contemplar esses aspectos.

Todos esses fatores devem ser ponderados ao analisar o fato de que os dados aqui apresentados não dão suporte para uma eventual predominância de substantivos em relação a verbos em crianças de diferentes idades em fase inicial de aquisição de vocabulário, ao menos na situação em que a pesquisa foi conduzida. O que os dados aqui apresentados e o conjunto de pesquisas produzidas sobre o tema parecem sugerir é que o desenvolvimento e a composição do vocabulário nas crianças são processos multideterminados, e ainda são necessárias investigações adicionais para se compreender, não apenas quais são os seus fatores componentes, mas também como ocorre a complexa interação entre eles.

\section{Referências}

Barrett, M. (1997). Desenvolvimento lexical inicial. In P. Fletcher \& B. Mcwhinney (Eds.), Compêndio da linguagem da criança (pp. 299-321). Porto Alegre, RS: Artes Médicas.

Bassano, D. (2000). Early development of nouns and verbs in French: Exploring the interface between lexicon and grammar. Journal of Child Language, 27, 521-559.

Bastos, J. C., Ramos, A. P. F., \& Marques, J. (2004). Estudo do vocabulário infantil: Limitações das metodologias tradicionais de coleta. Revista da Sociedade Brasileira de Fonoaudiologia, 9, 1-9.

Befi-Lopes, D. M., Cáceres, A. M., \& Araújo, K. (2007). Aquisição de verbos em pré-escolares falantes do português brasileiro. Revista CEFAC, 9, 444-452.

Bornstein, M. H., Cote, L. R., Maital, S., Painter, K., Park, S. Y., Pascual, L., ...Vyt, A. (2004). Cross-linguistic analysis of vocabulary in young children: Spanish, Dutch, French, Hebrew, Italian, Korean, and American English. Child Development, 75, 1115-1139.

Brown, R. (1973). A first language the early stages. Cambridge, MA: Harvard University Press.

Caselli, M. C., Bates, E., Casadio, P., Fenson, J., Fenson, L., Sanderl, L., \& Weir, J. (1995). A crosslinguistic study of early lexical development. Cognitive Development, 10, 159-199.

Choi, S. (2000). Caregiver input in English and Korean: Use of nouns and verbs in book-reading and toy-play contexts. Journal of Child Language, 27, 69-96.

Choi, S., \& Gopnik, A. (1995). Early acquisition of verbs in Korean: A cross-linguistic study. Journal of Child Language, 22, 497-529.

Frankenburg, W. K., Dodds. J., Archer, P., Shapiro, H., \& Bresnick, B. (1992). The Denver II: A major revision and restandardization of the Denver Developmental Screening Test. Pediatrics, 89, 91-97.

Gelman, S. A., \& Tardif, T. (1998). Acquisition of nouns and verbs in Mandarin and English. In E. Clark (Ed.), The proceedings of the twenty-ninth annual child language research forum (pp. 27-36). Stanford, UK: Center for the Study of Language and Information.

Gentner, D. (1982). Why nouns are learned before verbs: Linguistic relativity versus natural partitioning. In S. A. Kuczaj II (Ed.), Language development: Vol. 2. Language, thought, and culture (pp. 301-334). Hillsdale, NJ: Lawrence Erlbaum. 
Schmidt, A., Costa, A. R. C., Norberto, M. C. \& Voss, A. (2014). Ocorrência de Classes Gramaticais na Fala Espontânea de Crianças de 18 a 41 Meses com Pares.

Goldfield, B. A. (1993). Noun bias in maternal speech to oneyear-olds. Journal of Child Language, 20, 85-99.

Golinkoff, R. M., \& Hirsh-Pasek, K. (2006). Introduction: Progress on the verb learning front. In K. Hirsh-Pasek \& R. M. Golinkoff (Eds.), Action meets word: How children learn verbs (pp. 3-28). New York: Oxford University Press.

Gopnik, A., \& Choi, S. (1995). Names, relational words, and cognitive development in English and Korean-speakers: Nouns are not always learned before verbs. In M. Tomasello \& W. Merriman (Eds.), Beyond names for things: Young children's acquisition of verbs (pp. 63-80). Hillsdale, NJ: Erlbaum.

Imai, M., Li, L., Haryu, E., Okada, H., Hirsh-Pasek, K., Golinkoff, R. M., \& Shigematsu, J. (2008). Novel noun and verb learning in Chinese-, English-, and Japanese-speaking children. Child Development, 79, 979-1000.

Jackson-Maldonado, D., Thai, D., Marchman, V., Bates, E., \& Gutierrez-Clellen, V. (1993). Early lexical development in Spanish-speaking infants and toddlers. Journal of Child Language, 20, 523-549.

Kauschke, C., \& Hofmeister, C. (2002). Early lexical development in German: A study on vocabulary growth and vocabulary composition during the second and third year of life. Journal of Child Language, 29, 735-757.

Kim, M., McGregor, K. K., \& Thompson, C. K. (2000). Early lexical development in English- and Korean-speaking children: Language-general and language-specific patterns. Journal of Child Language, 27, 225-254.

Mogford, K., \& Bishop, D. V. M. (2002). Desenvolvimento da linguagem em condições normais. In D. V. M. Bishop \& K. Mogford (Eds.), Desenvolvimento da linguagem em circunstâncias excepcionais (pp. 1-26). Rio de Janeiro, RJ: Revinter.

Muszkat, M., \& Mello, C. B. (2009). Neurodesenvolvimento e linguagem. In T. Barbosa, C. C. Rodrigues, \& C. B. Mello (Eds.), Temas em dislexia (pp. 1-15). Porto Alegre, RS: Artes Médicas.

Nelson, K. (2007). Young minds in social worlds. Cambridge, MA: Harvard University Press.

Ogura, T., Dale, P. S., Yamashita, Y., Murase, T., \& Mahieu, A. (2006). The use of nouns and verbs by Japanese children and their caregivers in book-reading and toy-playing contexts. Journal of Child Language, 33, 1-29.

Pessoa, L. F., \& Seidl de Moura, M. L. (2011). Fala materna dirigida à criança em cenários comunicativos específicos: Um estudo longitudinal. Psicologia: Teoria e Pesquisa, 27, 439-447.

Pine, J. M., Lieven, E. V. M., \& Rowland, C. (1996). Observational and checklist measures of vocabulary composition: What do they mean? Journal of Child Language, 23, 573-590.

Scherer, S., \& Souza, A. P. R. (2011). Types e tokens na aquisição típica de linguagem por sujeitos de 18 a 32 meses falantes do português brasileiro. Revista CEFAC, 13, 838-845.

Stoll, S., Bickel, B., Lieven, E., Paudyal, N. P., Banjade, G., Bhatta, T. N., ...Rai, N. K. (2012). Nouns and verbs in Chintang: Children's usage and surrounding adult speech. Journal of Child Language, 39, 284-321.

Tardif, T. (1996). Nouns are not always learned before verbs: Evidence from Mandarin speaker's early vocabularies. Developmental Psychology, 32, 492-504.

Tardif, T., Gelman, S. A., \& Xu, F. (1999). Putting the "noun bias" in context: A comparison of English and Mandarin. Child Development, 70, 620-635.

Tomasello, M. (1999). The cultural origins of human cognition. Cambridge, MA: Harvard University Press.
Tomasello, M. (2003). Constructing a language: A usage-based theory of language acquisition. Cambridge, MA: Harvard University Press.

Vidor, D. C. G. M. (2008). Aquisição lexical inicial por crianças falantes de português brasileiro: Discussão do fenômeno da explosão do vocabulário e da atuação da hipótese do viés nominal (Tese de doutorado, Pontifícia Universidade Católica do Rio Grande do Sul, Porto Alegre, RS, Brasil).
Recebido: 05/07/2012

$1^{a}$ revisão: 05/11/2012

$2^{a}$ revisão: 05/02/2013

Aceite final: 13/03/2013 